\title{
METHODOLOGY FOR THE DESIGN OF FILTERS AND DIGITAL CONTROLLERS IMPLEMENTED ON EMBEDDED SYSTEMS
}

\section{MIGUEL PÉREZ PEREIRA, HOLMAN MONTIEL ARIZA, EDWAR JACINTO GÓMEZ}

Universidad Distrital Francisco José de Caldas, Facultad Tecnológica,Bogotá, Colombia

\begin{abstract}
This research shows a design and implementation methodology for filters and digital controllers that can be implemented in any general purpose embedded system and even those systems that base their processor on low cost microcontroller type embedded systems, this methodology clearly shows how crucial maximum values such as sampling frequency are found $f_{s}$, cut-off frequency $f_{c}$ The STM32F401 is a digital filter with a digital controller, which can be easily migrated to a highly complex digital controller, This is done taking into account that when designing a digital filter and/or a digital controller you have a wide spectrum of applications in different branches of engineering, but a big step is taken for real implementations in real time digital controls applied to robotics. It is well known that when high order filter and/or controller orders are needed digital designs are far ahead of their analog counterparts; But all these advantages are linked to disadvantages in its implementation, even more when you want to implement solutions that work in real time, for applications where you cannot use an embedded system of specific purpose as are the DSP's (Digital Signal Processor) due to its great economic cost, and must opt for general purpose and low cost embedded microcontroller type systems, as a viable alternative for their developments. These devices, although more affordable in cost, have lower performance features that compromise the operation of the system, limiting the sampling frequency and its computational capacity to perform high order filters and controllers.

KEYWORDS: Digital Controllers, Embedded Systems, Real-Time Systems, STM32, High-Performance Mcus, Digital Filters
\end{abstract}

Received: Oct 08, 2020; Accepted: Oct 28, 2020; Published: Nov 12, 2020; Paper Id.: IJMPERDOCT202047

\section{INTRODUCTION}

A digital filter and a digital controller are discrete Linear and Time Invariant (LTI) systems used to extract characteristics from the frequency domain on sampled signals (Andrés \& Quiroz, 2017)in which, you can have a fixed or variable sampling frequency (Kuruppu \& Shibilski, 2018)depending on your specific application, this extraction is done by means of a difference equation that describes the system, implementing these systems on hardware and working in real time forces at first the use of hardware devices with processors specifically designed to perform calculations of successive additions and multiplications efficiently and quickly, Such devices are known as specific purpose processors and are called DSPs (Jaiswal et al., 2018)(Mudgal et al., 2018), the main disadvantage of this hardware is its high economic cost and complexity of its programming (since each DSP has its own development platform, and sometimes the designer must program in the corresponding assembly language of the manufacturer), which makes it a not very viable alternative for systems that intend to keep its cost low; in addition to the devices mentioned above, programmable FPGA(Das et al., 2016)type devices can be used, which for this type of applications have a high performance but their programming language and lack of tools, make their 
implementation difficult, in addition to the fact that their cost-benefit ratio is not the best for low-budget applications.

Other hardware that can be used are the embedded systems of general purpose type microcontroller (Varshney \& Tiwari, 2018), which abound in the market and are of low cost, such systems are becoming viable alternatives for the implementation of systems that work in real time because they have suffered in the last times an increase in their computational speeds and performance, sample of it are the STM32 processors (Lei \& Sa, 2020), being one of the most economical on the market, have a double precision floating point unit and a digital-analog converter that allows sampling rates appropriate to the real-time systems used and another without number of applications with similar characteristics (Kendall et al., 2019)to the digital filters implemented in this work(Jongsataporn \& Cheevapreecha, 2020), in addition to being able to work in robotics applications where digital controllers are made in real time and multitask(Song et al., 2017)(Yu et al., 2017)(Yuan et al., 2016)(Engin et al., 2016)(Shirai et al., 2016)(R. J. \& Mohana, 2020).

Taking into account the above, the main objective of this work is to present a design methodology for filters and digital controllers that work in real time (FDTR) (Rodrigues et al., 2018)(Mudgal et al., 2018), which can be implemented in any general purpose embedded system, even those based on microcontrollers, such design methodology, shows how the maximum system breakpoints are calculated for the critical parameters $\mathrm{Fs}, \mathrm{Fc}$, and order of equations in differences with a natural implementation type (FIR)(Joshi \& Skhurge, 2017)(Singh \& Potnis, 2018)and infinite impulse response (IIR) (Gnanaprakasam \& Chitra, 2017)(Lesnikov et al., 2018). In addition, this design methodology (Lesnikov et al., 2019)will be tested by implementing two filters, one FIR and another IIR, in the embedded system STM32F401 of the company STMicroelectronics where its effectiveness and simplicity will be shown. All these tests can be taken quickly and simply to implement different types of digital controllers of different complexity and order.

\section{MATERIALS AND METHODS}

The development of this document initially contemplates the description of the Digital FIR filters, IIR and digital controllers, as well as its equations, in addition it explains the Digital Filter in real time, its main characteristics and particularities, later it shows how the critical points of the filters and controllers implemented in any embedded system are found and finally it shows the implementation of the FIR and IIR filters in the Embedded System STM32F401.

\section{Digital Filters}

A Digital Filter or a digital controller, can be described as a system in which a discrete $\mathrm{X}(\mathrm{n})$ signal enters and operates on it to produce a new $\mathrm{Y}(\mathrm{n})$ output signal, this transformation occurs due to the interaction between the input and the $\mathrm{H}(\mathrm{n})$ Transfer Function, this interaction extracts characteristics from the frequency domain to a signal in the discrete time domain. The basic operation of a filter and / or digital controller is relatively simple, since they use equations in differences to transform the input signal, according to their response, digital filters and in general any system can be classified into two categories, the first are those whose Pulse Response is Finite (FIR) and the second are those whose Pulse Response is Infinite (IIR)(Lesnikov et al., 2019)

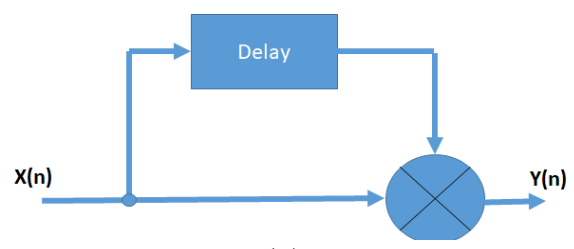

(a) 


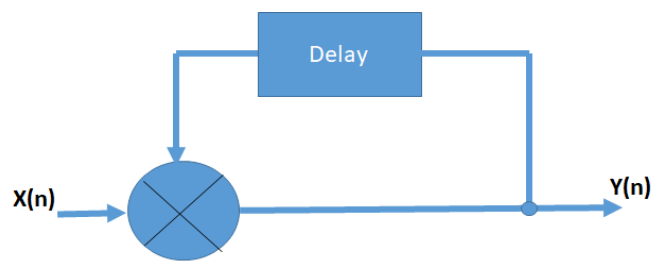

(b)

Figure 1: Blocks diagram; (a) FIR system, (b) IIR system

A FIR system shown in Figure 1(a), which, as its name indicates, are those systems that respond finitely to an input impulse signal, these systems are called systems without feedback, which is their main characteristic and makes them highly stable, an FIR filter is a system without feedback that responds in its transfer function to the following equation.

$$
\begin{gathered}
Y(n)=\sum_{i=0}^{c} b_{i} x(n-i)(1) \\
H(Z)=b_{0} Z+b_{1} Z^{-1}+\cdots . .+b_{c} Z^{-c}(2)
\end{gathered}
$$

Equation 1 shows the output equation of an order FIR filter $\boldsymbol{c}$ where $\boldsymbol{x}(\boldsymbol{n}-\boldsymbol{i})$ represent the system inputs in each time period that are added up to find the output, and $\boldsymbol{b}_{\boldsymbol{i}}$ represents the filter coefficients, a better known version of this equation can be seen in 2, which represents the transfer function of the filter [20], and results from applying the transform $Z$ to the above equations.

Now, Figure 1(b) shows the basic block diagram of an IIR system, which is the basis for the IIR filters, as you can see, these systems have feedback which makes them conducive to instability, the IIR filters are governed by the following equations.

Equation 3 represents the output of a FIR Filter of order $c$ In this equation you can see that the output of the system depends on both the inputs $X(n-i)$ in each time period, as well as from the outputs $Y(n-i)$. Equation 4 shows the transfer function of the system after applying the $Z$ transform.

$$
\begin{gathered}
Y(n)=\sum_{i=0}^{c} b_{i} x(n-i)+\sum_{j=0}^{c} a_{j} y(n-j) \\
H(Z)=\frac{b_{0} Z+b_{1} Z^{-1}+\cdots . .+b_{c} Z^{-c}}{a_{0} Z+a_{1} Z^{-1}+\cdots . .+a_{c} Z^{-c}}
\end{gathered}
$$

Equation 5 shows the formula for a FIR filter that can be implemented in any embedded system and results from applying the inverse $\mathrm{Z}$ transform to equation 2, equation 6 does the same with equation 4 and represents the equation to be implemented in an IIR Filter.

$$
\begin{gathered}
Y(n)=b_{0} X(n)+b_{1} X(n-1)+\cdots+b_{c} X(n-c)(5) \\
Y(n)=b_{0} X(n)+b_{1} X(n-1)+\ldots+b_{c} X(n-c)-b_{0} Y(n)-b_{1} Y(n-1)-\ldots-b_{c} Y(n-c)(6)
\end{gathered}
$$

\section{METHODOLOGY OF DESIGNING}

To begin with the methodology of designing an FDTR, it must be taken into account that it is a system capable of 
synchronizing with the physical process from which you want to extract information through any of its filtering operations in a dynamic, correct, and limited in time (Mudgal et al., 2018). This means that a filter and digital controller can process a signal that comes from the real world while respecting the time considerations of the system. Now, to process digitally a signal coming from the real world and return it to the real world implies the use of analog-digital and analog-digital conversion hardware that spend time, such time would be added to the system processing time, which further complicates the problem, figure 2 shows the block diagram of an FDTR.

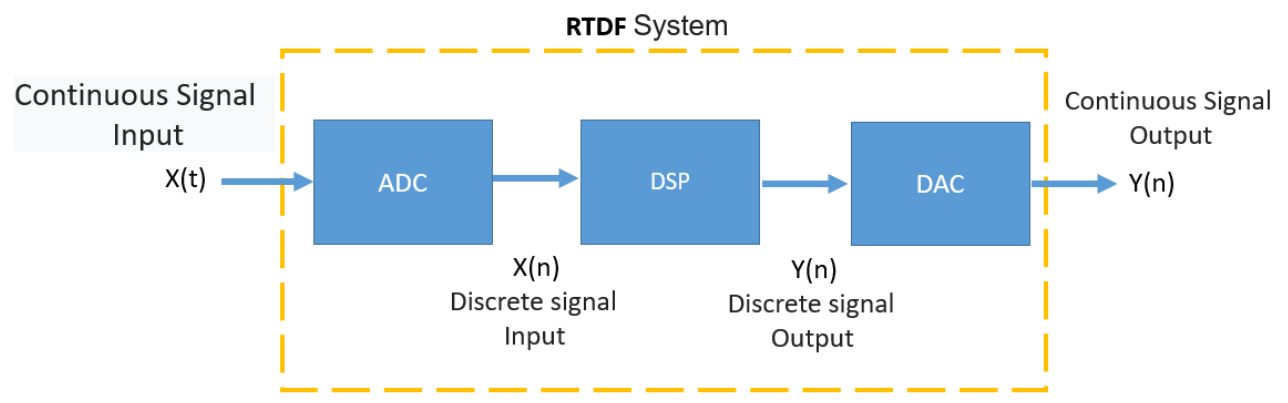

Figure 2: General block diagram of an FDTR

An FDTR consists of three fundamental components (ADC, Processor, DAC) which interact with each other to achieve the expected processing and thus deliver the signal to the real world. These three components are crucial for the calculation of system time, which is essential for any system that seeks to work in real time.

\section{Steps for Designing the Filter and/or Controller}

In the following section, the methodology for the design and implementation of the FDTR will be presented. This methodology will be shown by means of numbered steps, so that the developer can implement them and reach a satisfactory result, it must be remembered that this methodology is applicable to any type (FIR, IIR), configuration or filter order.

In the first step you should measure the maximum processing time of the system, remember that the system consists of three elements the ADC, the Processor, DAC which contribute time to the maximum processing time. There are several methods to measure the time taken by these three sections, here are two proposed:

- Use the embedded systems own tools for time calculations, these tools are called Timer and are present in all embedded systems, an example of the use of this tool can be seen in the section of implementation of the filters.

- The second one is to use an external pin of the embedded system to count the time, it is used in the following way, when the ADC reading starts the pin is put in "1" logic and when the DAC measurement ends it is lowered to "0", this pin is measured with an oscilloscope and the pulse duration is determined, which is the sampling period $P_{S}$ of the system

It should be remembered that, although the filter has not been designed, the formula of the filter to be implemented should be set with any value in the coefficients, since the calculation time of the formula used by the processor contributes to the sampling period of the system.

Another aspect to take into account is that, although most of the embedded systems available in the market have $\mathrm{ADC}$, there are few that have DAC, as it is the case of the STM32F401 embedded system, when this happens, the DAC is 
replaced by the PWM module and a passive filter is added outside which is known as signal reconstruction filter.

Already having the sampling frequency calculated as the inverse of the sampling period $\left(f_{s}=\frac{1}{P_{S}}\right) \operatorname{In}$ order to calculate the coefficients of the filter to be implemented, we proceed to the calculation of the coefficients. Although there are different methods for the calculation of these coefficients, one of the most used and simplest is the use of the FDATOOL tool [5] and/or the SISOTOOL controller design tool of the MatLab® software for the calculation of these coefficients. Figure 3 shows an example of the calculation of the coefficients for a low pass FIR filter of order $3 f_{s}=48 K$, $f_{\text {pass }}=1 \mathrm{~K}, f_{\text {stop }}=1,5 \mathrm{~K}$ and an IIR filter of order 2 , with $f_{s}=48 \mathrm{k}$ y $f_{c}=1 \mathrm{~K}$.

Figure 3 shows the calculation of the coefficients for two classes of filters, in Figure 3(a) it can be seen that for FIR filters they only have coefficients in the numerator of the transfer function (see equation 2), Figure 3(b) shows the calculation for an IIR filter, which has coefficients in both the numerator and the denominator. These coefficients are implemented in equations 5 and 6 respectively.

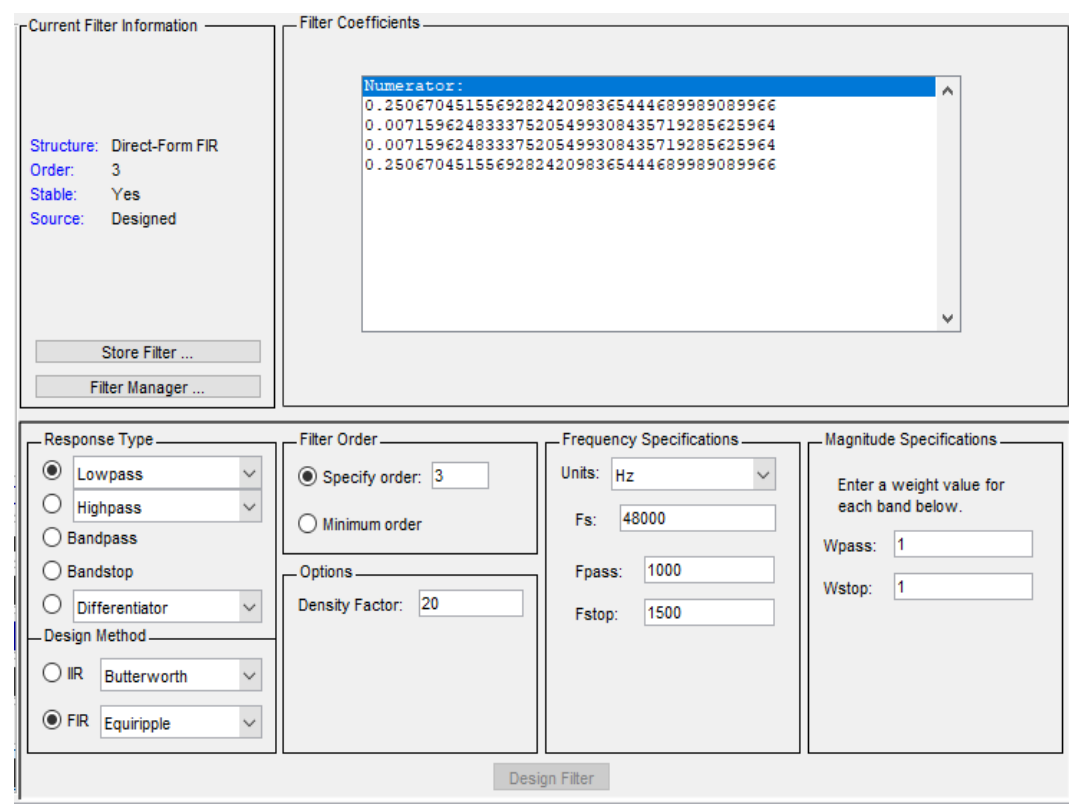

(a) 


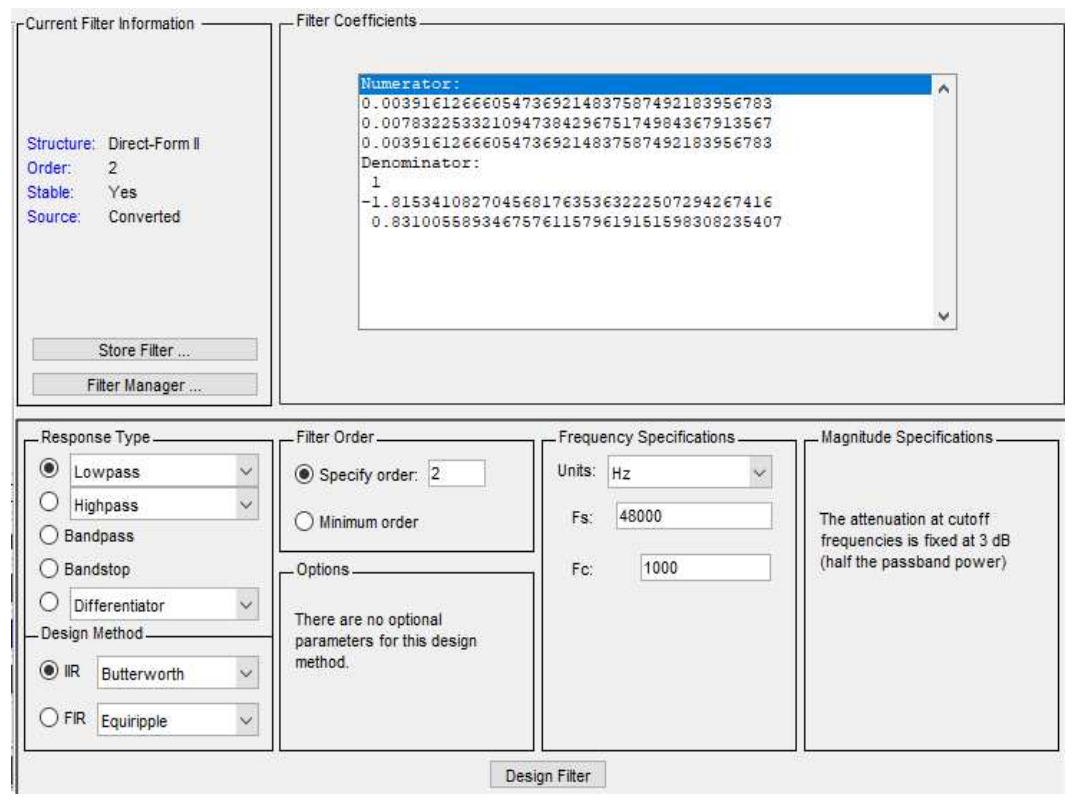

(b)

Figure 3: Design of filters with FDATOOL; (a) FIR, (b) IIR

The last step is the implementation of the algorithm in the embedded system, two examples of this in the STM32F401 embedded system can be seen in the following section.

\section{IMPLEMENTATION AND RESULTS}

In this section, the design methodology proposed in this research will be tested by implementing a FIR filter and an IIR filter in the embedded system STM32F401 of the company STMicroelectronics.

This microcontroller-type embedded system was selected because of its low cost, as it does not exceed 2 USD. It also works at $84 \mathrm{MHz}$ and 105 DMIPS, which is more than acceptable performance for digital filter and digital controller applications, as it has a double precision floating point unit enhanced for DSP applications, fully applicable to current realtime robotic solutions.

This type of technology of the STM32 processors are implemented in $90 \mathrm{~nm}$ technology, low power consumption technology, with the possibility of generating tasks in real time using a real-time operating system RTOS that makes when the processor is not performing information processing is put into a state of low power consumption, with a minimum consumption of $9 \mu \mathrm{A}$ in its deepest sleep mode.

One of the most attractive features of this specific processor is its flash memory of 512 Kbytes of flash memory and 96Kbytes of SRAM, which makes it applicable to highly complex filtering systems and robotics, with a great variety of tasks and/or memory usage for signal processing and at the end the feature that makes this processor more attractive is its analog to digital converter, which, being 2.4 mega samples per second makes the sampling delay minimum and opens a great spectrum to filtering applications and high performance controller design.

For this research, a Kaiser Low Pass FIR filter will be implemented, $F_{c}=1000 \mathrm{~Hz}$ and order of 12 , and a Butterworth IIR low pass filter with $F_{c}=1000 \mathrm{~Hz}$ and order 6 , these two filters were chosen because they have the same number of operations to implement in the equation, remember that implementing low-pass filters, high-pass filters or other 
configurations does not affect the procedure, only the H(n) coefficients change. Now let's start testing the steps of the methodology.

\section{Measurement of Maximum System Processing Time}

To calculate this maximum time, a timer or Systick was used, which is an internal timer that is present in all ARM Cortex M microcontrollers.

This tool does not consume processor resources since it is an independent hardware peripheral. With this tool, the time spent by the system between reading the ADC, calculating the equation, and writing to the PWM, for the two types of filters to be implemented, is calculated. The equations to implement are shown in equation 7 and 8 .

For the proposed FIR filter the equation is:

$$
Y(n)=b_{0} X(n)+b_{1} X(n-1)+\cdots+b_{12} X(n-12)(7)
$$

And for the IIR filter the equation is:

$$
Y(n)=\frac{b_{1}}{a_{0}} X(n)+\frac{b_{2}}{a_{0}} X(n-1)+\ldots+\frac{b_{6}}{a_{0}} X(n-6)-\frac{a_{1}}{a_{0}} Y(n)-\frac{a_{2}}{a_{0}} Y(n-1)-\ldots-\frac{a_{6}}{a_{0}} Y(n-6)(8)
$$

The time measurements are displayed using a telnet type serial console, for two filters the same values of provisional coefficients, this so that the time comparison does not depend on the value of these.

\section{Calculation of $F_{s}$ Maximum for the Two Types of Filters}

Samplingperiods were found with the above procedure, for the FIR filter the sampling period is 26us which gives us a $F_{s}=38,4 \mathrm{KHz}$ and for the IIR filter the sampling period is 21 us which gives us a $F_{s}=47,6 \mathrm{KHz}$. Since the provisional coefficients of the two filters were the same, plus the ADC and PWM do not change, the difference in sampling frequency is assumed as a particular result of the calculation of the equation by the processor and the floating-point unit.

\section{Calculation of the Filter Coefficients}

As explained above, although there are different ways to calculate the filter coefficients, one of the easiest is to use MatLab's® FDATOOL tool, Figure 4 shows the calculation of these coefficients, for the two selected filters.

Figure 4(a) shows the design of the FIR filter, in the graph of this filter you can see the high curl that has the system in the attenuated frequencies, this curl is one of the disadvantages of these filters for low orders, but this is compensated by the linearity of its phase, positive point for these. Now, Figure 4(b) shows the design for the IIR filter, as can be seen in the graph, its response is better with respect to the FIR, but this results in phase alignment. 


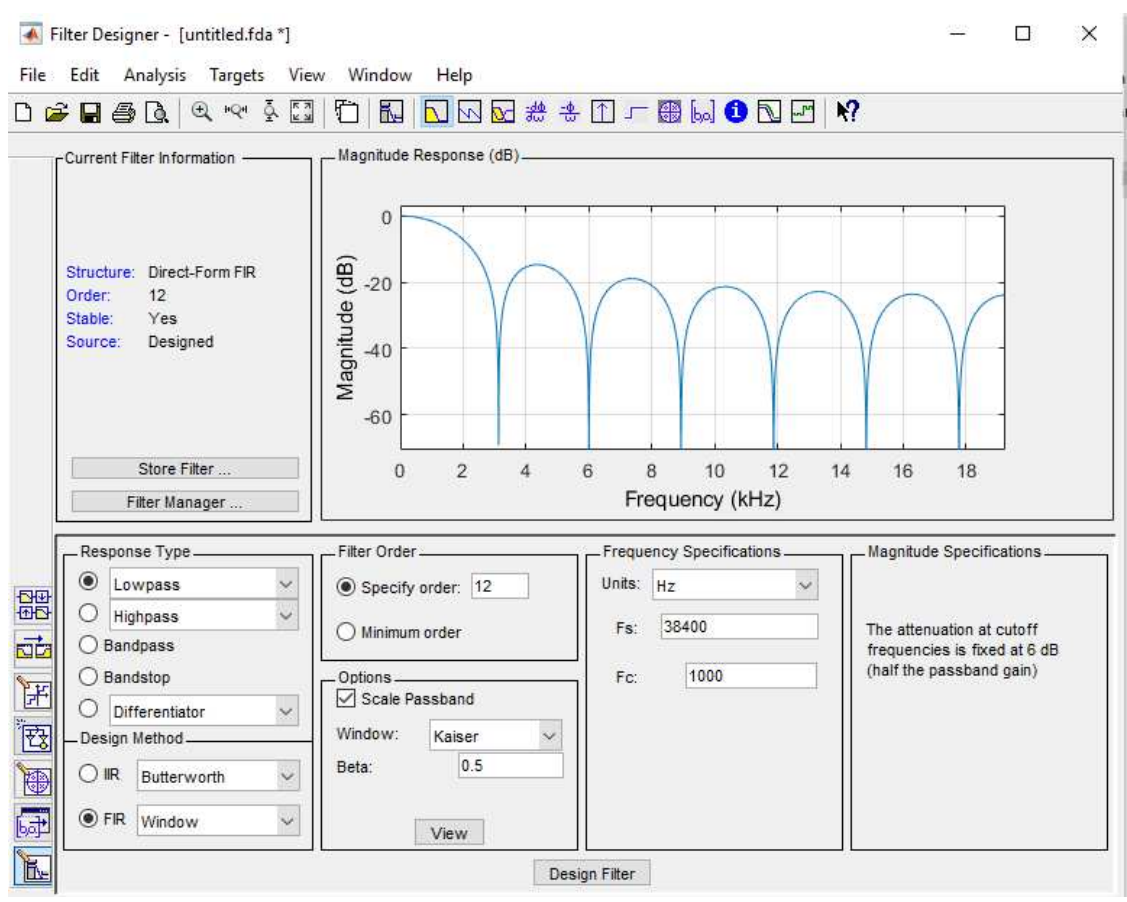

(a)

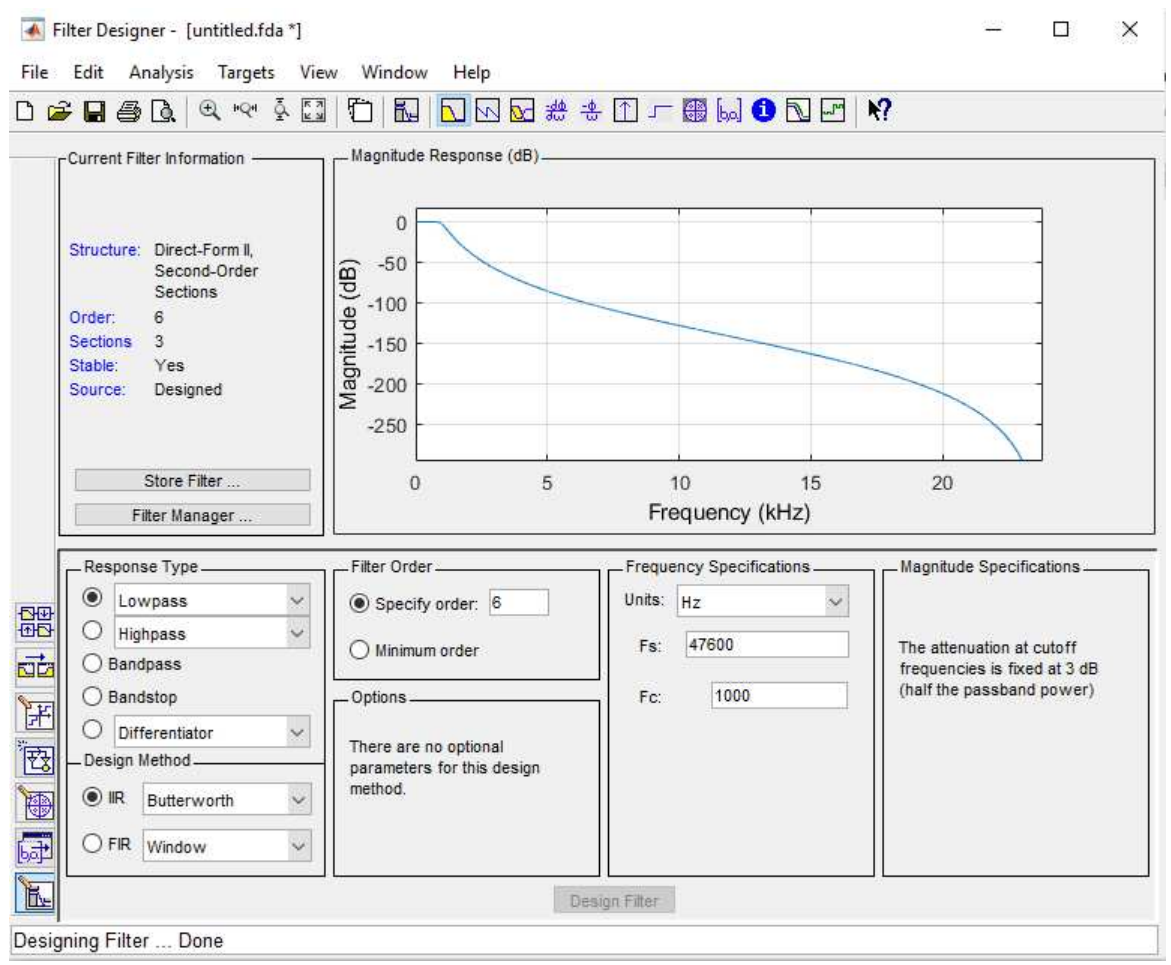

(b)

Figure 4: Filter design with FDATOOL; (a) FIR, (b) IIR.

\section{Results}

Since this embedded system does not have DAC, first the PWM, the frequency of the PWM, had to be configured $F_{p w m}$

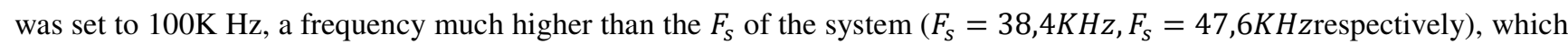


is essential for its operation, since if the $F_{p w m}$ is less than the $F_{S}$ If you do not have a signal, this system will not be able to reconstruct the output signal. To reconstruct the signal and to be able to observe it in the oscilloscope, a RC filter was implemented at the output of the embedded system.

The reconstruction filter that was implemented, the $F_{c}$ of this filter was designed at $10.6 \mathrm{KHz}$ frequency more than enough to reconstruct the waves coming from the implemented filters (since these filters have $F_{c}=1 \mathrm{kHz}$ ), the importance of this filter is to remove $F_{p w m}$ which is $100 \mathrm{kHZ}$, the output signals of the implemented filters can be seen in Figure 6 .

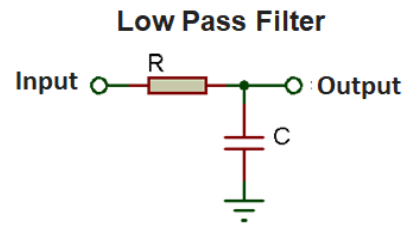

$$
F_{c}=\frac{1}{2 \pi R C}=\frac{1}{(2 \pi)(15 K \Omega)(1 \eta f)}=10.61 K H z
$$

\section{Figure 5: Signal Reconstruction Filter}

Figure 6a shows the response of the implemented FIR filter. $V_{p p}=1,4 \mathrm{v}$ output, which corresponds to $70 \%$ of the input voltage the system cut-off frequency is $F_{c}=1075 \mathrm{HZIn}$ the figure $6 \mathrm{~b}$, the output of the IIR filter is shown, it is very close to the $1 \mathrm{KHz}$ cutoff frequency with which we designed it. $V_{p p}=1,4 \mathrm{~V}$ output, the system cut-off frequency is $F_{c}=$ 1136HZThe filter is farther away than the FIR filter, but equally close to the $F_{c}$ with which it was designed.

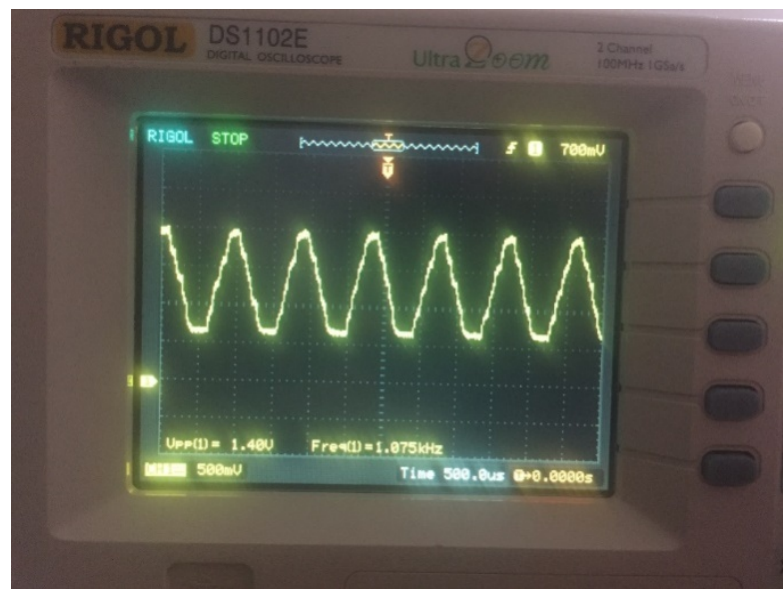

(a)

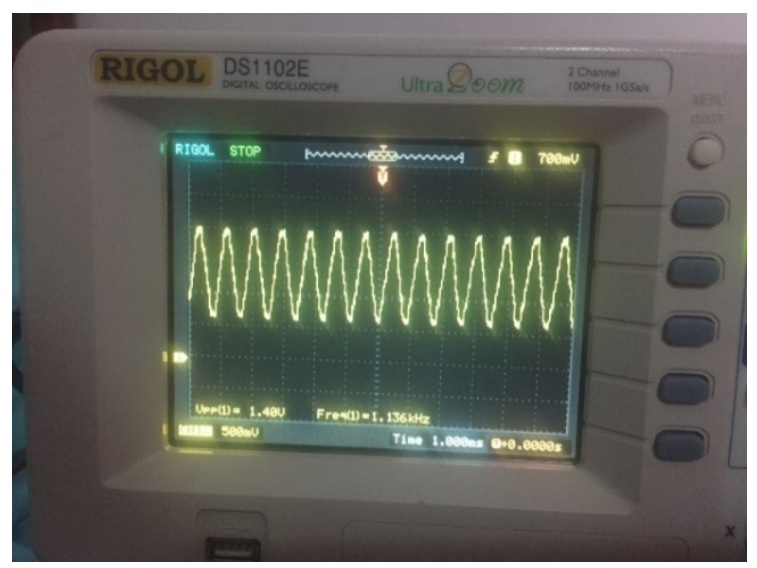

(b)

Figure 6: Response graph of implemented filters; (a) FIR, (b) IIR. 


\section{CONCLUSIONS}

Designing FTDR and implementing them on low-cost embedded systems has become a necessity for many developers. This research demonstrates that this implementation is possible and also proposes a design methodology that consists of only 4 steps to achieve it, In addition to high order digital controllers applicable to autonomous mobile robotics, as demonstrated in the document, it is important to highlight that in this research the design methodology was raised and also tested on the embedded system STM32F401 of the company STMicroelectronics.

Regarding the implementation of these filters, the following aspects can be highlighted. Designing FIR filters and IIR filters presents the same complexities, since their main difference in design is based on the H(n) to be implemented, while in FIR filters the transfer function only has terms in the numerator, for IIR filters they have terms in both the numerator and the denominator of the transfer function, which leads to a change of signs in the equation to be implemented.

The $F_{s}$ found in the two implemented systems was $F_{s}=38,4 \mathrm{KHz}$ for FIR filters of order 12 and $F_{s}=47,6 \mathrm{KHz}$ for IIR filters of order 6 , this shows that using low-cost embedded system is possible and functional, since the bandwidth presented by these systems is sufficient for countless applications, even those applications that work with non-audible frequencies, it should be noted that this sampling frequency is adequate and sufficient for all applications of autonomous mobile robotics stand-alone type of today, since for such applications require sampling periods never exceeding $20 \mathrm{KHz}$ and orders never exceeding 6 , in terms of the complexity of the equation in differences used.

The FDTR designed by means of this methodology, can be implemented with very little margin of error, since they were designed at a $F_{c}=1 \mathrm{~K}$ and in its implementation gave $F_{c}=1,075 \mathrm{KHz}$ and $F_{c}=1,136 \mathrm{KHz}$, respectively, which corresponds to an error of $6.9 \%$ in the case of FIR and $11.9 \%$ in the case of IIR, very negligible errors for this type of system, moreover these errors can be corrected by avoiding the truncation of data in the processor. Since the processor has a double precision floating point unit, it is possible to implement controllers with coefficients equal to those designed in a desktop computer, without losing any degree of precision or requiring truncation that could generate errors in the behavior of the filters or controllers designed.

The use of the PWM module to replace the DAC for those embedded systems that do not have it, becomes a real alternative, since the speed of this module allows its use without any inconvenience, just add an external passive filter to the embedded system for the reconstruction of the signal, which does not present any challenge or inconvenience.

\section{REFERENCES}

1. Andrés, F., \& Quiroz, R. (2017). Metodología de Diseño de Filtros Digitales Basada en Estrategias de Optimización Metaheurística.

2. Das, R., Foundation, S. K., Guha, A., Tech, S. B., Foundation, S. K., Bhattacharya, A., Tech, S. B., \& Foundation, S. K. (2016). FPGA Based Higher Order FIR Filter Using. International Conference on Signal Processing, Communication, Power and Embedded System (SCOPES), 111-115.

3. Engin, M. (2016). Embedded and real time system design: A case study fire fighting robot. 2016 5th Mediterranean Conference on Embedded Computing (MECO), Bar, 18-21. doi: 10.1109/MECO.2016.7525712.

4. Gnanaprakasam, C. N., \& Chitra, K. (2017). Soft-computing based digital filter design to analyze vibration signals of induction motor. 2017 International Conference on Algorithms, Methodology, Models and Applications in Emerging 
Technologies, ICAMMAET 2017, 2017-Janua, 1-4. https://doi.org/10.1109/ICAMMAET.2017.8186627

5. Jaiswal, R. K., Bagre, M., \& Mishra, R. A. (2018). Performance evaluation of digital and RNS based filter for fast DSP processors. 2017 International Conference on Emerging Trends in Computing and Communication Technologies, ICETCCT 2017, 2018-Janua(2), 1-4. https://doi.org/10.1109/ICETCCT.2017.8280309

6. Jongsataporn, T., \& Cheevapreecha, S. (2020). An Implementation of Digital Parametric Equalizer on STM32 Processor. 2 , 113-117. https://doi.org/10.1109/ecti-con49241.2020.9158299

7. Joshi, P. C., \& Skhurge, D. (2017). Comparative analysis of embedded computing platforms For FIR filter implementation. Proceedings - 2nd International Conference on Computing, Communication, Control and Automation, ICCUBEA 2016. https://doi.org/10.1109/ICCUBEA.2016.7860122

8. Kendall, B., Sawyer, R. J., Chang, H., Cook, N., Wild, J., Mo, T. E., Rochberg, F., Sherman, L. W., Nelson, R. R., Dosi, G., Nelson, R. R., Dosi, G., Pyka, A., Nelson, R. R., Pyka, A., Saviotti, P. P., Nelson, R. R., Bhrigu K Lahkar, Prize, D. D., ... Persians, A. (2019). Embedded electronic scale measuring system based on STM32 single chip microcomputer. Foresight, 23(3), 1-9.

9. Kuruppu, S. S., \& Shibilski, A. (2018). Clock Variation Impact on Digital Filter Performance. 2018 9th IEEE Annual Ubiquitous Computing, Electronics and Mobile Communication Conference, UEMCON 2018, 340-344. https://doi.org/10.1109/UEMCON.2018.8796816

10. Lei, J., \& Sa, J. (2020). Design and implementation of a single-phase bipolar spwm inverter power supply based on stm32. Proceedings - 2020 3rd International Conference on Advanced Electronic Materials, Computers and Software Engineering, AEMCSE 2020, 641-644. https://doi.org/10.1109/AEMCSE50948.2020.00140

11. Lesnikov, V., Naumovich, T., \& Chastikov, A. (2018). Number-theoretical analysis of the structures of classical IIR digital filters. 2018 7th Mediterranean Conference on Embedded Computing, MECO 2018 - Including ECYPS 2018, Proceedings, June, 1-4. https://doi.org/10.1109/MECO.2018.8406099

12. Lesnikov, V., Naumovich, T., \& Chastikov, A. (2019). The Topography of a Third Order IIR Digital Filter Zeros and Poles in the z-Plane Discretized due to the Quantization of the Direct Form Coefficients. 2019 8th Mediterranean Conference on Embedded Computing, MECO 2019 - Proceedings, June, 1-4. https://doi.org/10.1109/MECO.2019.8760068

13. Mudgal, E., Mukuntharaj, S., Modak, U., \& Rao, Y. S. (2018). Template Based Real-Time Speech Recognition Using Digital Filters on DSP-TMS320F28335. Proceedings - 2018 4th International Conference on Computing, Communication Control and Automation, ICCUBEA 2018, 1-6. https://doi.org/10.1109/ICCUBEA.2018.8697848

14. R. J. Franklin \& Mohana. (2020). Industry 4.0: Real Time Embedded System with Integrated Data Acquisition. 2020 Third International Conference on Smart Systems and Inventive Technology (ICSSIT), Tirunelveli, India, 637-642. doi: 10.1109/ICSSIT48917.2020.9214251.

15. Rodrigues, N. M., Janeiro, F. M., \& Ramos, P. M. (2018). Digital filter performance for zero crossing detection in power quality embedded measurement systems. I2MTC 2018 - 2018 IEEE International Instrumentation and Measurement Technology Conference: Discovering New Horizons in Instrumentation and Measurement, Proceedings, 1-6. https://doi.org/10.1109/12MTC.2018.8409701

16. Shirai, T., Osawa, K., Chishiro, H., Yamasaki, N. \& Inaba, M. (2016). Design and Implementation of a High Power Robot Distributed Control System on Dependable Responsive Multithreaded Processor (D-RMTP). 2016 IEEE 4th International Conference on Cyber-Physical Systems, Networks, and Applications (CPSNA), Nagoya, 19-24. doi: 10.1109/CPSNA.2016.12 
17. Singh, N., \& Potnis, A. (2018). A review of different optimization algorithms for a linear phase FIR filter. International Conference on Recent Innovations in Signal Processing and Embedded Systems, RISE 2017, 2018-Janua(4), 44-48. https://doi.org/10.1109/RISE.2017.8378122

18. Song, H., Popovics, J., \& Park, J. (2017). Contactless ultrasonic wavefield imaging of concrete elements using an automated scanning MEMS ultrasonic sensor array. IEEE International Ultrasonics Symposium, IUS, 1-3. https://doi.org/10.1109/ULTSYM.2017.8092489

19. Varshney, V., \& Tiwari, M. (2018). Realization of an FIR filter using ATMEGA32 microcontroller. 2017 International Conference on Emerging Trends in Computing and Communication Technologies, ICETCCT 2017, 2018-Janua(2), 1-4. https://doi.org/10.1109/ICETCCT.2017.8280325

20. Yu, C., Ma, X., Fang, Qian, Yao, S. \& Zou, Y. (2017). Design of controller system for industrial robot based on RTOS Xenomai. 2017 12th IEEE Conference on Industrial Electronics and Applications (ICIEA), Siem Reap, 221-226. doi: 10.1109/ICIEA.2017.8282846.

21. Yuan, C., Ghamry, K. A., Liu, Z., Zhang, Y., \& Member, S. (2016). Unmanned Aerial Vehicle Based Forest Fire Monitoring and Detection Using Image Processing Technique. 2016 IEEE Chinese Guidance, Navigation and Control Conference (CGNCC), 1870-1875. https://doi.org/10.1109/CGNCC.2016.7829074 